\title{
ON LEPTONIC DECAY OF ORIENTED HEAVY LEPTON WITH MASSLESS SPIN $3 / 2$ NEUTRINO
}

I. OTS. ORIENTEERITUD RASKE LEPTONI LEPTONLAGUNEMISEST MASSITA 3/2-SPINNIGA NEUTRIINO KORRAL

И. ОТС. О ЛЕПТОННОМ РАСПАДЕ ОРИЕНТИРОВАННОГО ТЯЖЕЛОГО ЛЕПТОНА ПРИ БЕЗМАССОВОМ НЕИТРИНО СО СПИНОМ $3 / 2$

(Presented by H. Keres)

In previous papers $\left[{ }^{1-3}\right]$ the decays

$$
\begin{aligned}
& L^{-} \rightarrow l^{-}+\tilde{v}_{l}+v_{L}, \\
& L^{+} \rightarrow l^{+}+v_{l}+\tilde{v}_{L}
\end{aligned}
$$

were discussed with $3 / 2$ for the spin of decaying lepton and with spin $1 / 2$ for all the other leptons. In this paper the same decay process is considered with the only difference that now both the heavy lepton $(L)$ and its neutrino $v_{L}$ are taken to be of spin $3 / 2$ particles. Serious difficulties exist in describing zero mass higher spin particles, however, they can be avoided assuming that only the «gauge invariant» spinor

$$
u_{\mu v}=q_{\mu} u_{v}-q_{v} u_{\mu}
$$

appears in matrix elements [ $\left.{ }^{4}\right]$. Then, when summing over helicity $\pm 3 / 2$ states as required for massless particles, the projection operator is given by

$$
\begin{gathered}
\sum_{s= \pm 3 / 2} u_{\mu v}(q, s) \bar{u}_{\rho \sigma}(q, s)=(\hat{q} / 2 m)\left[q_{\mu} q_{\rho}\left(-g_{v \sigma}+\gamma_{v} \gamma_{\sigma} / 2\right)-\right. \\
-q_{\mu} q_{\sigma}\left(-g_{v \rho}+\gamma_{v} \gamma_{\rho} / 2\right)-q_{v} q_{\rho}\left(-g_{\mu \sigma}+\gamma_{\mu} \gamma_{\sigma} / 2\right)+ \\
\left.+q_{v} q_{\sigma}\left(-g_{\mu \rho}+\gamma_{\mu} \gamma_{\rho} / 2\right)\right] .
\end{gathered}
$$

According to our assumption the coupling of $v_{L}$ must proceed through the spinor curl $v_{\mu v}^{L}=\partial_{\mu} v_{v}^{L}-\partial_{v} v_{\mu}^{L}$, and the interaction Lagrangian for reactions (1) in the local case with $V-A l$ lepton current may be written as

$$
\Omega=\frac{G}{\sqrt{2} M_{L}}\left[\bar{l} \gamma^{\mu}\left(1-\gamma_{5}\right) v_{l}\right]\left[\bar{v}_{\mu v}^{L}\left(1-\alpha \gamma_{5}\right) L^{v}\right]+\text { h.c. },
$$

where $M_{L}$, the $L$ mass, is introduced so that $G$ is of the same, dimension 
as the Fermi coupling constant. In our calculations we also take into account the intermediate vector boson corrections to the decay parameters, modifying them by terms of the order $\omega=M_{L}^{2} / M_{W}^{2} \quad\left(M_{W}-\right.$ mass of the intermediate boson).

The energy-angular distribution of final $l$ leptons in the leptonic decay of oriented spin $3 / 2$ heavy leptons with spin $3 / 2 v_{L}$ may be given formally in the same way as in the case of spin $1 / 2 v_{L}\left[{ }^{3}\right]$. Thus, in the rest frame of decaying lepton we have

$$
\begin{aligned}
d W \mp & =\frac{G^{2} M_{L}^{5}}{24(2 \pi)^{4}} \int d \Omega \int_{0}^{1 / 2}\left[\tilde{F}_{0}(x, \alpha) \pm F_{1}(x, \alpha) t_{i} n^{i}+\right. \\
& \left.+\widetilde{F}_{2}(x, \alpha) t_{i j} n^{i} n^{j} \pm \widetilde{F}_{3}(x, \alpha) t_{i j k} n^{i} n^{j} n^{k}\right] d x
\end{aligned}
$$

where

$$
F_{i}(x, \alpha)=F_{i}(x, \alpha)+\omega F_{i}^{\prime}(x, \alpha) .
$$

But now the invariant functions $F_{i}(x, \alpha)$ and $F_{i}^{\prime}(x, \alpha)$ are given as follows:

$$
\begin{aligned}
F_{0}(x, \alpha) & =2 x^{2}\left[\left(1+\alpha^{2}\right)\left(7 / 16-4 x / 3+9 x^{2} / 8-11 x^{3} / 30\right)+\right. \\
& \left.+2 \alpha\left(1 / 16-x / 3+13 x^{2} / 24-3 x^{3} / 10\right)\right] \\
F_{1}(x, \alpha) & =\left(3 x^{2} / 5\right)\left[\left(1+\alpha^{2}\right)\left(11 / 24-7 x / 6+3 x^{2} / 20+3 x^{3} / 5\right)+\right. \\
& \left.+2 \alpha\left(29 / 24-13 x / 6-49 x^{2} / 60+11 x^{3} / 15\right)\right] \\
F_{2}(x, \alpha) & =\left(3 x^{2} / 20\right)\left[\left(1+\alpha^{2}\right)\left(23 x / 6-39 x^{2} / 4+11 x^{3} / 3\right)+\right. \\
& \left.+2 \alpha\left(17 x / 6-83 x^{2} / 12+3 x^{3}\right)\right] \\
F_{3}(x, \alpha)= & \left(3 x^{2} / 160\right)\left[\left(1+\alpha^{2}\right)\left(21 x^{2}-36 x^{3}\right)+2 \alpha\left(19 x^{2}-44 x^{3}\right)\right] \\
F_{0}^{\prime}(x, \alpha)= & 2 x^{3}\left[\left(1+\alpha^{2}\right)\left(1 / 3-29 x / 24+41 x^{2} / 30-8 x^{3} / 15\right)+\right. \\
& \left.+2 \alpha\left(1 / 12-11 x / 24+4 x^{2} / 5-7 x^{3} / 15\right)\right] \\
F_{1}^{\prime}(x, \alpha) & =\left(3 x^{3} / 5\right)\left[\left(1+\alpha^{2}\right)\left(5 / 12-61 x / 60-2 x^{2} / 15+14 x^{3} / 15\right)+\right. \\
& \left.+2 \alpha\left(5 / 12-19 x / 60-23 x^{2} / 15+16 x^{3} / 15\right)\right] \\
F_{2}^{\prime}(x, \alpha) & =\left(x^{3} / 20\right)\left[\left(1+\alpha^{2}\right)\left(-13 / 4+79 x / 4-35 x^{2}+16 x^{3}\right)+\right. \\
& \left.+2 \alpha\left(-7 / 4+61 x / 4-30 x^{2}+14 x^{3}\right)\right] \\
F_{3}^{\prime}(x, \alpha) & =\left(3 x^{4} / 160\right)\left[\left(1+\alpha^{2}\right)\left(-9+48 x-56 x^{2}\right)+\right. \\
& \left.+2 \alpha\left(-11+52 x-64 x^{2}\right)\right] .
\end{aligned}
$$

All notations used in this paper can be found in $\left[{ }^{1,3}\right]$.

\section{REFERENCES}

1. Ot s, I., Preprint F-9, Tartu, 1979.

2. О т с И., Изв. АН ЭССР, Физ, Матем., 28, № 2, 155-157 (1979).

3. Ots, I., ENSV TA Toim., Füüs. Matem., 29, № I, $97-100$ (1980).

4. Alles, W., D ra g o n i, M., IFUB 78-12, Bologna, 1978.

Academy of Sciences of the Estonian SSR, Institute of Physics 